ARTICLE

Received 16 Aug 2015 | Accepted 23 Nov 2015 | Published 18 Jan 2016

DOI: $10.1038 /$ ncomms10276

OPEN

\title{
Termination layer compensated tunnelling magnetoresistance in ferrimagnetic Heusler compounds with high perpendicular magnetic anisotropy
}

Jaewoo Jeong ${ }^{1, \star}$, Yari Ferrante ${ }^{1,2, \star}$, Sergey V. Faleev ${ }^{1}$, Mahesh G. Samant ${ }^{1}$, Claudia Felser $^{3}$ \& Stuart S.P. Parkin ${ }^{1,2}$

Although high-tunnelling spin polarization has been observed in soft, ferromagnetic, and predicted for hard, ferrimagnetic Heusler materials, there has been no experimental observation to date of high-tunnelling magnetoresistance in the latter. Here we report the preparation of highly textured, polycrystalline $\mathrm{Mn}_{3} \mathrm{Ge}$ films on amorphous substrates, with very high magnetic anisotropy fields exceeding $7 \mathrm{~T}$, making them technologically relevant. However, the small and negative tunnelling magnetoresistance that we find is attributed to predominant tunnelling from the lower moment $\mathrm{Mn}-\mathrm{Ge}$ termination layers that are oppositely magnetized to the higher moment $\mathrm{Mn}-\mathrm{Mn}$ layers. The net spin polarization of the current reflects the different proportions of the two distinct termination layers and their associated tunnelling matrix elements that result from inevitable atomic scale roughness. We show that by engineering the spin polarization of the two termination layers to be of the same sign, even though these layers are oppositely magnetized, high-tunnelling magnetoresistance is possible.

\footnotetext{
${ }^{1}$ IBM Almaden Research Center, San Jose, California 95120, USA. ${ }^{2}$ Max-Planck Institute for Microstructure Physics, 06120 Halle (Saale), Germany. ${ }^{3}$ Max-Planck Institute for Chemical Physics of Solids, 01187 Dresden, Germany. * These authors contributed equally to this work. Correspondence and requests for materials should be addressed to S.S.P.P. (email: stuart.parkin@mpi-halle.mpg.de).
} 
K ey to the successful development of magnetic random access memory (MRAM), one of the most promising emerging non-volatile memory technologies today, are new magnetic materials for the magnetic tunnel junction (MTJ) memory elements that have sufficient stability against thermal fluctuations to sustain deeply scaled devices. The most promising magnetic materials to date are considered to be magnetic alloys formed from $\mathrm{Co}, \mathrm{Fe}$ and $\mathrm{B}$, in conjunction with $\mathrm{MgO}(001)$ tunnel barriers ${ }^{1-3}$. The magnetic electrodes must possess sufficient perpendicular magnetic anisotropy (PMA) that their magnetizations lie perpendicular to the plane of the MTJ device, since this allows for reduced currents to switch the magnetization of the electrode that forms the memory layer of the device using spin torque ${ }^{3,4}$. The PMA of $\mathrm{Co}-\mathrm{Fe}-\mathrm{B}$ layers arises from the interfaces between these layers and the tunnel barrier and/or the underlayer on which the Co-Fe-B layer is deposited. Thus, these layers must be made sufficiently thin that the interface PMA overcomes the demagnetization energy that arises from the magnetic volume and increases in proportion with the magnetic volume of the $\mathrm{Co}-\mathrm{Fe}-\mathrm{B}$ layer. In practice, this means that the PMA is too weak to overcome thermal fluctuations when the device has a critical dimension $<\sim 20 \mathrm{~nm}$ in size, since the thickness of the magnetic layer has to be below that required to maintain its moment perpendicular, and, below that needed to switch the magnetic layer with reasonable current densities. Magnetic materials in which the PMA is derived from volume magnetocrystalline anisotropy are then needed. One of the most promising class of such materials are the Heusler alloys-compounds having the chemical formula $X_{2} Y Z$ or X'X' $Y Z$, wherein X, X', X' and Y are transition metals, or lanthanides (rare-earth metals), and $\mathrm{Z}$ is from a main group metal ${ }^{5}$. Some of these compounds are ferromagnetic or ferrimagnetic depending on the exchange interaction between the magnetic moments on the $\mathrm{X}$ and/or $\mathrm{Y}$ sites. Moreover, while the parent Heusler compounds are cubic and exhibit weak or no significant magnetic anisotropy, the structure of some of these compounds is found to be tetragonally distorted: due to this distortion the magnetization exhibited by these compounds may be aligned along the tetragonal axis. Thus, thin films formed from such materials may exhibit PMA due to a magnetocrystalline anisotropy associated with the tetragonally distorted structure. Some examples of such tetragonal Heusler compounds are $\mathrm{Mn}_{3-x} \mathrm{Ga}$ (ref. 6) and $\mathrm{Mn}_{3} \mathrm{Ge}$ (ref. 7).

Thin films of these materials have been shown to exhibit large PMA but, to date, all work on these materials has involved films that are grown epitaxially on single crystalline substrates such as $\mathrm{SrTiO}_{3}(001)$ or $\mathrm{MgO}(001)$ using seed layers formed from a variety of materials but preferably $\mathrm{Cr}$ or Pt (refs 7-11). Such single crystalline substrates are not useful for MRAM applications in which the MTJs must be deposited on wires formed from polycrystalline copper, which may be covered with other layers that are also polycrystalline or amorphous.

Here we show that thin films of ferrimagnetic $\mathrm{Mn}_{3} \mathrm{Ge}$ with giant PMA can be grown on amorphous substrates $\left(\mathrm{Si}(001) / \mathrm{SiO}_{2}\right)$ using underlayers formed from $\mathrm{TaN} / \mathrm{IrMn}_{3}$ (TI). The magnetic properties of these films are comparable or superior to films that we have grown under similar conditions on single crystal $\mathrm{MgO}(001)$ substrates using epitaxial $\mathrm{Cr}(001)$ underlayers. Very high magnetic anisotropy fields exceeding $7 \mathrm{~T}$ are found. However, the tunnelling magnetoresistance (TMR) is negative and much smaller than theoretical predictions for tunnel junction devices formed with $\mathrm{MgO}$ tunnel barriers. We attribute this to predominant tunnelling from the lower moment $\mathrm{Mn}-\mathrm{Ge}$ termination layers that are oppositely magnetized to the higher moment $\mathrm{Mn}-\mathrm{Mn}$ layers. The net spin polarization of the current reflects the different proportions of the two distinct termination layers and their associated tunnelling matrix elements at the tunnel barrier interface, that result from inevitable atomic scale roughness. We have confirmed that a second perpendicularly magnetized Heusler compound, $\mathrm{Mn}_{2} \mathrm{CuSb}$, which we identified by computational materials discovery methods, similarly displays small TMR, but that the sign of the spin polarization of each of the termination layers is opposite to that of their magnetization, in contrast to $\mathrm{Mn}_{3} \mathrm{Ge}$. Therefore, the low TMR that we have found in $\mathrm{Mn}_{3} \mathrm{Ge}$ and $\mathrm{Mn}_{2} \mathrm{CuSb}$, which we attribute to termination layer compensation, is not an inherent property of ferrimagnetic Heuslers. The spin polarization of the two termination layers can be engineered to be of the same sign even though these layers are oppositely magnetized, therefore providing a path to low magnetization electrodes with high TMR for high density spin transfer torque MRAM applications.

\section{Results}

Growth of highly textured $\mathrm{Mn}_{3} \mathrm{Ge}$ on amorphous substrates. Highly textured, polycrystalline and tetragonal $\mathrm{Mn}_{3} \mathrm{Ge}$ films were grown by either ion-beam deposition or d.c.-magnetron sputtering in an ultra-high vacuum chamber with a base pressure of $\sim 4 \times 10^{-10}$ torr on $\mathrm{Si}(001)$ substrates covered with $250 \AA$ of amorphous $\mathrm{SiO}_{2}$. We find that seed layers formed from bilayers of $\mathrm{TaN} / \mathrm{IrMn}_{3}$ that are first deposited on the $\mathrm{SiO}_{2}$ induce (001) textured $\mathrm{Mn}_{3} \mathrm{Ge}$ films that are tetragonally distorted. $\mathrm{IrMn}_{3}$ is known to have a $\mathrm{L1}_{2}$ structure that is cubic, and which matches one of the sublattices of the structure that $\mathrm{Mn}_{3} \mathrm{Ge}$ is known to form. Moreover, the lattice mismatch between $\mathrm{IrMn}_{3}$ and $\mathrm{Mn}_{3} \mathrm{Ge}$ is $<1 \%$ (ref. 12). The $\mathrm{TaN}$ layer that is grown by reactive sputtering, promotes the growth of (001) textured $\mathrm{IrMn}_{3}$, since fcc- $\mathrm{IrMn}_{3}$ favours the (111) out-of-plane orientation when grown directly on amorphous $\mathrm{SiO}_{2}$ surface (Supplementary Fig. 1 and Supplementary Note 1). However, in contrast to theoretical predictions of giant values of TMR ${ }^{6,13,14}$ for MTJs using $\mathrm{Mn}_{3} \mathrm{Ge}$ electrodes we find much smaller values experimentally, which we attribute to compensation in the tunnelling spin current polarization from atomic layer variations of the electrode surface termination at the tunnel barrier interface. We propose that this is an inevitable consequence of ferrimagnets with layer-by-layer alternation of magnetization, when the spin polarization of these layers compensates each other. We confirm this conjecture by preparing MTJs using another ferrimagnetic Heusler compound, $\mathrm{Mn}_{2} \mathrm{CuSb}$, which also shows high PMA but yet very small TMR.

The structural and magnetic properties of the $\mathrm{Mn}_{3} \mathrm{Ge}$ films depend sensitively on its composition and atomic order. The latter is strongly influenced by the deposition temperature and subsequent anneal conditions, which also affect the smoothness of the $\mathrm{Mn}_{3} \mathrm{Ge}$ film. To achieve optimal MTJ performance the electrode must be atomically smooth. We find that the root mean square (r.m.s.) roughness of the $\mathrm{Mn}_{3} \mathrm{Ge}$ film, $r_{\text {r.m.s. }}$, measured using atomic force microscopy, increases significantly when the growth temperature $\left(T_{\mathrm{G}}\right)$ exceeds modest temperatures of just $\sim 200^{\circ} \mathrm{C}$, but higher growth temperatures are needed to sustain the Heusler structure, as measured from X-ray diffraction (Supplementary Fig. 2 and Supplementary Note 2). Thus, we find that an optimal growth method, which includes a three-step process for the $\mathrm{Mn}_{3} \mathrm{Ge}$ electrode, in which an initial $20 \AA \mathrm{Mn}_{3} \mathrm{Ge}$ layer is grown at $450^{\circ} \mathrm{C}$, followed by a thicker $\mathrm{Mn}_{3} \mathrm{Ge}$ layer deposited at $T_{\mathrm{G}}=150^{\circ} \mathrm{C}$, with a final in situ anneal at $450^{\circ} \mathrm{C}$ for $1-2 \mathrm{~h}$ in vacuum, gives smooth films $\left(r_{\text {r.m.s. }} \sim 3 \AA\right)$ with high PMA. During the annealing step of $\mathrm{Mn}_{3} \mathrm{Ge}$ films, there is a substantial inter-diffusion between $\mathrm{IrMn}_{3}$ and $\mathrm{Mn}_{3} \mathrm{Ge}$ layers (Supplementary Fig. 3), which causes deterioration of the magnetic properties of $\mathrm{Mn}_{3} \mathrm{Ge}$. We discovered that this 
interdiffusion can be prevented by using a thin $10-20 \AA$ TaN barrier between $\mathrm{IrMn}_{3}$ and $\mathrm{Mn}_{3}$ Ge layers, as revealed by electron energy loss spectroscopy measurements (Supplementary Note 3). Thus, the preferred underlayer is formed from $\mathrm{TaN} / \mathrm{IrMn}_{3} / \mathrm{TaN}$ (TIT). We note that a single TaN underlayer gives much poorer quality $\mathrm{Mn}_{3} \mathrm{Ge}$ layers.

Figure 1 compares the magnetic properties of $\mathrm{Mn}_{3} \mathrm{Ge}$ films with varying thickness grown on amorphous substrates using the three-step process and on a crystalline $\mathrm{MgO}(001)$ substrate with a $\mathrm{Cr}$ seed layer (MC). Excellent PMA is observed in all cases but the highest coercive and anisotropy fields are found for structures grown on the TIT underlayer. Coercive fields of $6 \mathrm{~T}$ and anisotropy fields exceeding $7 \mathrm{~T}$ are found. Figure $1 \mathrm{c}$ summarizes the magnetic moment $m$, coercivity $H_{\mathrm{C}}$ and uniaxial magnetic anisotropy $K_{\mathrm{U}}$ for these films. Values of $m$ for $\mathrm{Mn}_{3}$ Ge films grown on TIT underlayers are close to those theoretically predicted for bulk $\mathrm{Mn}_{3} \mathrm{Ge}$ (ref. 15), but $m$ is significantly lowered by $\sim 15-35 \%$ for $\mathrm{Mn}_{3} \mathrm{Ge}$ films grown using TI underlayers or a $\mathrm{MC}$ single crystal substrate. For the latter film, we also find that the magnetic anisotropy $K_{\mathrm{U}}$ is substantially lower: we attribute this to the large lattice mismatch $(\sim 7 \%)$ between $\mathrm{Cr}$ and $\mathrm{Mn}_{3} \mathrm{Ge}$.

Tunnelling magnetoresistance of $\mathbf{M n}_{3} \mathrm{Ge}$-based MTJ devices. MTJ devices were fabricated using standard lithographic techniques from film stacks whose structures are illustrated in Fig. 1b. The reference electrode was formed from the $\mathrm{Mn}_{3} \mathrm{Ge}$ Heusler compound, and the free electrode from an ultrathin layer of $\mathrm{CoFeB}$ with a composition of 20:60:20. Before patterning, these films were post-annealed at $350^{\circ} \mathrm{C}$ for $60 \mathrm{~min}$ in a high-vacuum chamber using an applied magnetic field of $1 \mathrm{~T}$ directed out of the plane of the sample. Devices with sizes of $1 \times 2 \mu \mathrm{m}^{2}$ and $\sim 30 \mathrm{~nm}$ in diameter were fabricated by optical lithography and e-beam lithography, respectively. Only the free layer was patterned to define the junction size while the reference layer was not patterned.

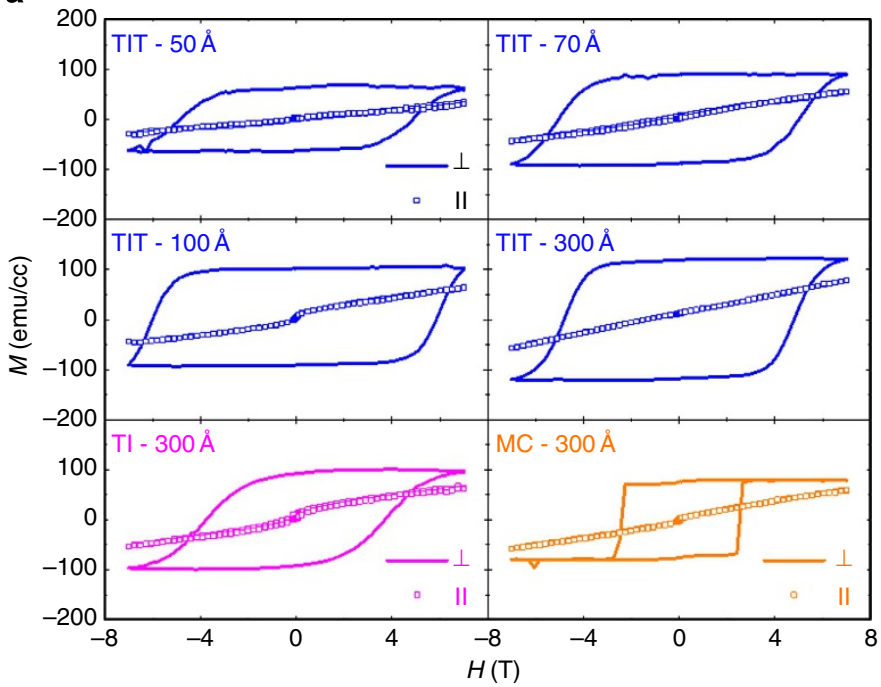

C

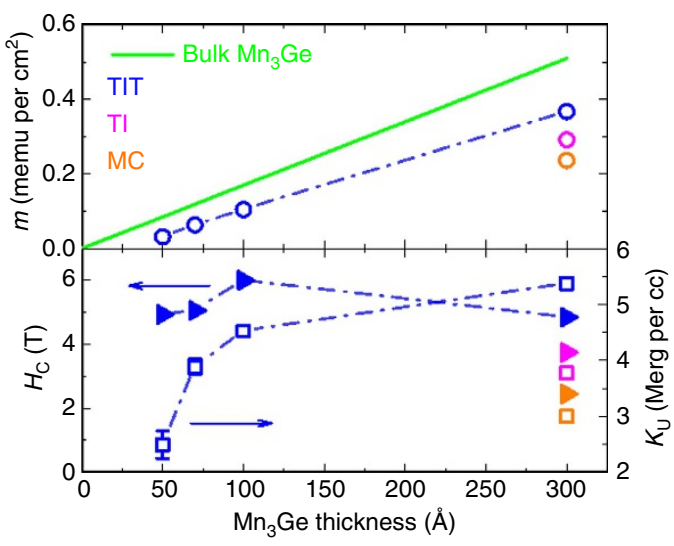

b

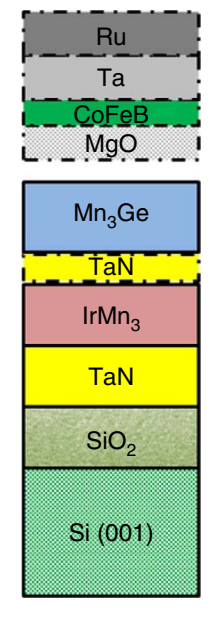

d

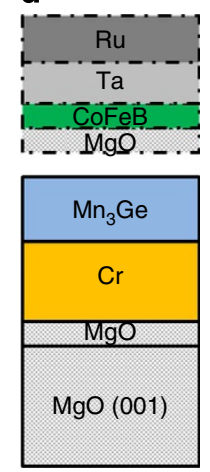

Figure 1 | $\mathbf{M n}_{\mathbf{3}} \mathrm{Ge}$ Heusler films with giant perpendicular magnetic anisotropy. (a) Magnetization versus field hysteresis loops of $\mathrm{Mn}_{3} \mathrm{Ge}$ films grown on $\mathrm{Si}(001) / \mathrm{SiO}_{2}$ substrate with $\mathrm{TI}$ (magenta) and TIT (blue) underlayers and $\mathrm{MgO}(001)$ substrate with $\mathrm{MC}$ (orange) underlayers. TI (TIT) have the following structure: $\mathrm{Si}(001) / \mathrm{SiO}_{2} / 200 \AA \mathrm{TaN} / 200 \AA \mathrm{IrMn}_{3}(/ 10 \AA \mathrm{TaN})$ while MC has the following structure: MgO(001)/20 $\AA \mathrm{MgO} / 400 \AA \mathrm{Cr}$. For the TIT films, the thickness of the $\mathrm{Mn}_{3} \mathrm{Ge}$ layer, deposited using the three-step process, was varied. Out of plane (in-plane) $M$ vs. $H$ loops are shown as solid lines (open squares). $\mathbf{b}$ and $\mathbf{d}$ are schematics of the MTJ structures grown on $\mathrm{Si}(001) / \mathrm{SiO}_{2}$ and $\mathrm{MgO}(001)$ substrates, respectively. In some cases a TaN diffusion barrier layer was used as indicated by a dashed line. For characterization of structural, topographical and magnetic properties of the $\mathrm{Mn}_{3} \mathrm{Ge}$ films, a $30 \AA \mathrm{Ta}$ film was used as a capping layer instead of the upper layers shown within the dotted lines. (c) Magnetic moment $m$, coercive field $H_{C}$ (solid triangles) and uniaxial magnetic anisotropy constant $K_{U}$ (empty squares), extrapolated from Fig. 1a, versus $\mathrm{Mn}_{3} \mathrm{Ge}$ thickness. The green straight line shows the calculated moment of bulk $\mathrm{DO}_{22}-\mathrm{Mn}_{3} \mathrm{Ge}$ (ref. 15). As shown in Fig. 1a, the $\mathrm{Mn}_{3} \mathrm{Ge}$ magnetization cannot be saturated in-plane using the available magnetic field (7 T); thus, $H_{\text {eff }}$ is a lower bound. 
a

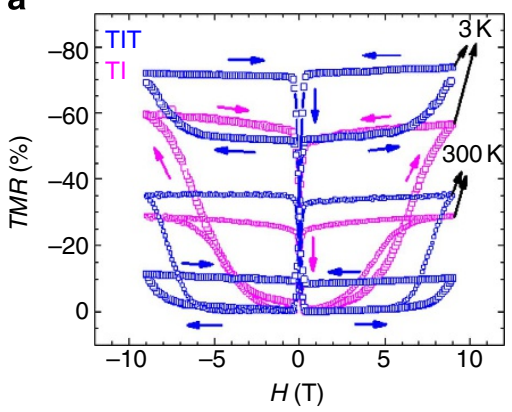

C

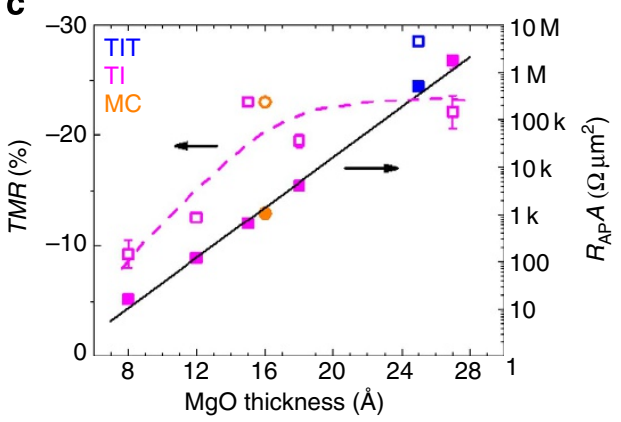

b

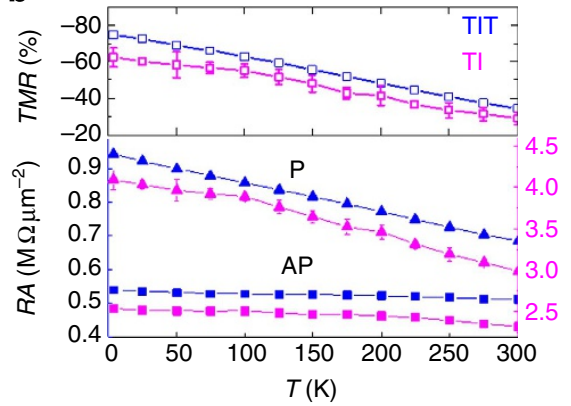

d

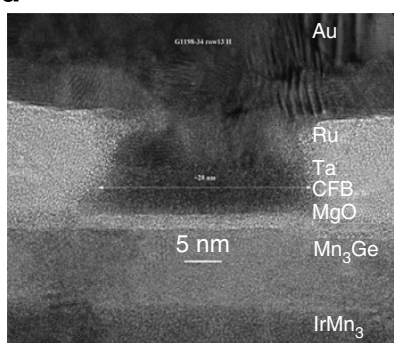

Figure 2 | Characteristics of $\mathbf{M n}_{\mathbf{3}} \mathbf{G e}$-based magnetic tunnel junctions. (a) TMR versus $H$ (perpendicular to the device) measured at $300 \mathrm{~K}$ (smaller squares) and $3 \mathrm{~K}$ (bigger squares) for MTJ devices grown using TI (magenta) and TIT (blue) underlayers. For the TIT junction, two sets of data were measured at $3 \mathrm{~K}$ (blue bigger squares) after cooling down the device from $300 \mathrm{~K}$ in a magnetic field of +9 and $-9 \mathrm{~T}$, respectively. These data are mirror images of each other, as can be seen in the figure. All the other measurements were performed without field cooling. (b) Temperature dependence of TMR, and $R_{\mathrm{P}} A$ and $R_{\mathrm{AP}} A$. (c) $\mathrm{MgO}$ thickness dependence of TMR (open symbols) and $R_{\mathrm{AP}} A$ product (solid symbols), averaged over $>20$ devices. Solid and dashed lines are guides to the eye for $R_{\mathrm{AP}} A$ and TMR, respectively. $R_{\mathrm{AP}} A$ scales exponentially with barrier thickness. (d) HRTEM image of an MTJ device $\sim 27 \mathrm{~nm}$ in size, with the structure: $\mathrm{Si} / 250 \AA \mathrm{SiO}_{2} / 200 \AA \mathrm{TaN} / 200 \AA \mathrm{IrMn}_{3} / 300 \AA \mathrm{Mn}_{3} \mathrm{Ge} / 15 \AA \mathrm{MgO} / 15 \AA \mathrm{CoFeB} / 50 \AA \mathrm{Ta} / 50 \AA \mathrm{Ru}$. HRTEM, high-resolution transmission electron microscopy.

Figure 2a compares TMR versus perpendicular magnetic field $H$ measured at $300 \mathrm{~K}$ (smaller squares) and $3 \mathrm{~K}$ (larger squares) for patterned MTJ devices $\left(1 \times 2 \mu \mathrm{m}^{2}\right)$ using TI and TIT underlayers. In each case high applied magnetic fields $( \pm 9 \mathrm{~T})$ are needed to align the magnetic moments of the $\mathrm{Mn}_{3} \mathrm{Ge}$ and $\mathrm{CoFeB}$ layers parallel to each other (P state) because of the giant uniaxial anisotropy of $\mathrm{Mn}_{3} \mathrm{Ge}$. The junction resistance is higher in the $\mathrm{P}$ state compared with the antiparallel (AP) state, obtained when the $\mathrm{CoFeB}$ moment switches close to zero field. Thus, the $\operatorname{TMR}\left(\left[\left(R_{\mathrm{AP}}-R_{\mathrm{P}}\right) / R_{\mathrm{AP}}\right] \times 100\right)$ is negative with values of $\sim-35 \%$ at $300 \mathrm{~K}$ and $\sim-74 \%$ at $3 \mathrm{~K}$, where $R_{\mathrm{P}}$ and $R_{\mathrm{AP}}$ are the junction resistances in the $\mathrm{P}$ and $\mathrm{AP}$ states, respectively. These are the highest values of TMR reported to date in perpendicularly magnetized MTJ devices using a tetragonally distorted Heusler compound as a magnetic electrode. Nonetheless, these values are much smaller than those predicted by density functional theory (DFT) calculations ${ }^{14}$, as discussed below.

For a given MTJ device $R_{\mathrm{AP}}$ barely changes, while $R_{\mathrm{P}}$ increases monotonically as $T$ decreases, resulting in higher TMR at low temperatures (Fig. 2b). These properties, as well as the dependence of the resistance-area product $R_{\mathrm{AP}} A$ and TMR on the barrier thickness (Fig. 2c) are characteristic of a high quality tunnel barrier. A cross-sectional high-resolution transmission electron microscopy image of a typical MTJ device with a width of $27 \mathrm{~nm}$, shown in Fig. 2d, illustrates the high quality of the structure and the device patterning.

Notwithstanding the exceptionally high PMA values exhibited by polycrystalline $\mathrm{Mn}_{3} \mathrm{Ge}$ films the surprisingly low TMR values lessens their potential importance for MTJ devices. We attribute the low TMR, as discussed below, to their ferrimagnetic structure. This limitation could be overcome by identifying Heusler compounds that display high PMA, and which are ferromagnetic.
Discovery of tetragonal Heuslers by computational methods. To identify potential candidate tetragonal materials we used computational materials discovery methods to calculate the structure and electronic properties of several hundred Heusler compounds (with $\mathrm{X}=\mathrm{Mn}, \mathrm{Fe}, \mathrm{Co}, \mathrm{Ni}, \mathrm{Cu}, \mathrm{Ru}, \mathrm{Rh}, \mathrm{Pd} ; \mathrm{Y}=\mathrm{Sc}$, $\mathrm{Mn}, \mathrm{Fe}, \mathrm{Co}, \mathrm{Ni}, \mathrm{Cu}, \mathrm{Ru}, \mathrm{Rh}, \mathrm{Pd}$, Os, Ir, Pt; and $\mathrm{Z}=\mathrm{Al}, \mathrm{Si}, \mathrm{Ga}, \mathrm{Ge}$, $\mathrm{In}, \mathrm{Sn}, \mathrm{Sb}$ ) for both the regular and inverse structures and for ferri- and ferromagnetic configurations. Surprisingly, $\sim 40 \%$ of these compounds are calculated to be tetragonal in their ground state. Rank ordering these tetragonal compounds according to the combination of the energy difference between the tetragonal and cubic structures and that between the inverse and regular structures, we identified $\sim 30$ possible candidate tetragonal materials, where the average energy difference exceeded $\sim 0.3 \mathrm{eV}$ per formula unit. Of these, we prepared eight in thin film form using the same buffer layers and deposition conditions as discussed above. Half of these compounds showed a tetragonal structure and two showed excellent PMA properties, namely, $\mathrm{Mn}_{2} \mathrm{CuSb}$ and $\mathrm{Rh}_{2} \mathrm{CoSb}$. In the other cases, we rationalize that the theoretical predictions were not fulfilled due to atomic disorder on the $\mathrm{X}$ and $\mathrm{Y}$ sites. This is particularly the case where $\mathrm{X}$ and $\mathrm{Y}$ have similar chemical properties or atomic size.

Similar magnetic properties were found for $\mathrm{Mn}_{2} \mathrm{CuSb}$ and $\mathrm{Rh}_{2} \mathrm{CoSb}$ films grown using either TI underlayers (Supplementary Fig. 4 and Supplementary Note 4 ) or an MC crystalline substrate but the latter films were typically smoother and better suited for MTJ studies. Results are shown in Fig. 3 for $\mathrm{Mn}_{2} \mathrm{CuSb}$. Square magnetic hysteresis loops consistent with PMA are found. The calculated lowest energy configuration of $\mathrm{Mn}_{2} \mathrm{CuSb}$ is a tetragonal ferromagnetic regular Heusler with a moment of $5.4 \mu_{\mathrm{B}}$ per formula unit (Table 1). Although we find that the deposited films are tetragonal (Supplementary Fig. 5 and Supplementary Note 5), 
in agreement with the calculations, the measured $c / a$ ratio is much smaller in the films $\left(c_{t} / \sqrt{2} a_{t} \sim 1.1\right)$ than that predicted for a fully ordered $\mathrm{Mn}_{2} \mathrm{CuSb}$ bulk compound $\left(c_{t} / \sqrt{2} a_{t} \sim 1.4\right)$, and thus only slightly distorted from the cubic phase. Moreover, the magnetization of the films is much too low- $\sim 0.4 \mu_{\mathrm{B}}$ per formula unit-to be consistent with the predicted ferromagnetic state, as is the TMR, as we discuss below. We rationalize these findings by the likelihood of chemical disorder within the deposited films. We note that the calculations predict that for the cubic phase the structure will be the inverse structure with a ferrimagnetic ordering, with a low moment (Table 1).

MTJ devices were prepared from $\mathrm{Mn}_{2} \mathrm{CuSb}$ films with the layer stack shown in Fig. 3d and the magnetic hysteresis loop shown in Fig. 3b. The latter shows clear independent switching of the free layer $(\mathrm{CoFeB})$ and the reference layer $\left(\mathrm{Mn}_{2} \mathrm{CuSb}\right)$ but TMR
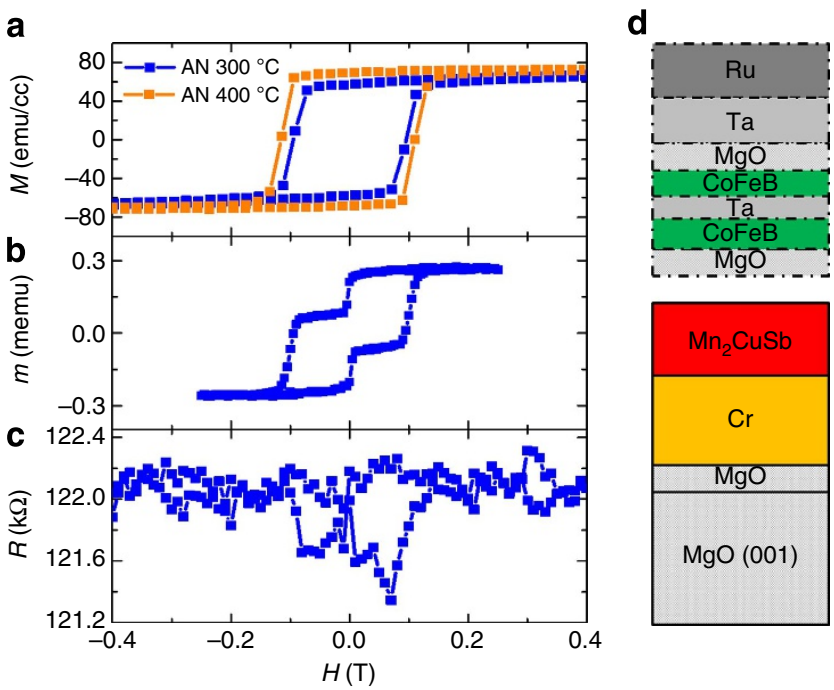

Figure 3 | Magnetics properties and TMR of $\mathbf{M n}_{2} \mathbf{C u S b}$ Heusler alloy.

(a) Out-of-plane $M$ versus $H$ hysteresis loops, at $300 \mathrm{~K}$, of $300 \AA$ thick $\mathrm{Mn}_{2} \mathrm{CuSb}$ films grown on $\mathrm{MC} \mathrm{MgO}(001)$ substrate at room temperature and post annealed at $300^{\circ} \mathrm{C}$ (blue) and $400^{\circ} \mathrm{C}$ (orange) for $30 \mathrm{~min}$. The anneal treatments were carried out in a high-vacuum anneal furnace, in an applied magnetic field of $1 \mathrm{~T}$ (out-of-plane direction) for $30 \mathrm{~min}$. (b) Out-ofplane $M$ vs. $H$ hysteresis loop of an MTJ film measured before lithography patterning. (c) Two-terminal junction $R$ vs. $H$ loop of a patterned MTJ device $\left(1 \times 2 \mu \mathrm{m}^{2}\right.$ size $)$. (d) Schematic of a $\mathrm{Mn}_{2} \mathrm{CuSb}$-based MTJ, with the structure: $\mathrm{MgO}(001) / 20 \AA \mathrm{MgO} / 400 \AA \mathrm{Cr} / 300 \AA \mathrm{Mn}_{2} \mathrm{CuSb} / 25 \AA \mathrm{MgO} /$ $14 \AA \mathrm{CoFeB} / 4 \AA \mathrm{Ta} / 7 \AA \mathrm{CoFeB} / 7 \AA \mathrm{MgO} / 50 \AA \mathrm{Ta} / 100 \AA \mathrm{Ru}$. The $\mathrm{Mn}_{2} \mathrm{CuSb}$ electrode was deposited at ambient temperature and in situ annealed at $300^{\circ} \mathrm{C}$ before the $\mathrm{MgO}$ barrier deposition. The entire stack was then post annealed at $325^{\circ} \mathrm{C}$ for $30 \mathrm{~min}$. curves measured on $1 \times 2 \mu \mathrm{m}^{2}$ size devices show only very small TMR values of $\sim-1 \%$ (Fig. $3 \mathrm{c}$ ).

Termination-layer dependent tunnelling spin polarization. We performed $a b$ initio calculations of the electronic structure and transport properties of $\mathrm{Mn}_{3} \mathrm{Ge} / \mathrm{MgO} / \mathrm{Fe}$ and $\mathrm{Mn}_{2} \mathrm{CuSb} / \mathrm{MgO} / \mathrm{Fe}$ MTJs (see Methods for details of the calculations). Note that we use bcc Fe rather than $\mathrm{CoFeB}$ that is used in the experiments to simplify the calculations. The TMR for $\mathrm{Mn}_{3} \mathrm{Ge} / \mathrm{MgO} / \mathrm{Fe}$ MTJs is shown in Fig. 4a as function of the number of $\mathrm{MgO}$ layers, $N_{\mathrm{MgO}}$, for $N_{\mathrm{MgO}} \geq 2$. Two definitions of TMR are shown: $\left(T_{\mathrm{P}}-T_{\mathrm{AP}}\right) / \min \left(T_{\mathrm{P}}, T_{\mathrm{AP}}\right)$, that can vary from $-\infty$ to $\infty$, (that we use for the experimental data) and $\left(T_{\mathrm{P}}-T_{\mathrm{AP}}\right) /\left(T_{\mathrm{P}}+T_{\mathrm{AP}}\right)$, that can vary from -1 to 1 . Here $T_{\mathrm{P}}$ and $T_{\mathrm{AP}}$ are the transmission functions (calculated at zero bias voltage) corresponding to the $\mathrm{P}$ and AP states, respectively. The most important result is that the TMR depends sensitively on the atomic configuration of the termination layer in the $\mathrm{Mn}_{3} \mathrm{Ge}$ adjacent to the $\mathrm{MgO}$ tunnel barrier. The calculation gives magnetic moments of +4.08 and $-3.12 \mu_{\mathrm{B}}$ for the $\mathrm{Mn}-\mathrm{Mn}$ and $\mathrm{Mn}-\mathrm{Ge}$ termination layers, respectively. The TMR has opposite signs for the two termination layers, $\mathrm{Mn}-\mathrm{Mn}$ and $\mathrm{Mn}-\mathrm{Ge}$, increasing in magnitude with $N_{\mathrm{MgO}}$ for $\mathrm{Mn}-\mathrm{Mn}$ but decreasing in magnitude for Mn-Ge. These results can be understood from the layer dependent, spin-dependent density of states calculated for the bulk electronic structure of $\mathrm{Mn}_{3} \mathrm{Ge}$, and the well understood symmetry spin-filtering properties of the $\mathrm{MgO} / \mathrm{Fe}$ interface. The spin polarization of the native termination layers is negative in both cases but much bigger for Mn-Ge (see Supplementary Fig. 6 and Supplementary Note 6 for more details). A Brillouin zone filtering effect ${ }^{16}$ arising from the $\mathrm{Mn}_{3} \mathrm{Ge} / \mathrm{MgO}$ interface tends to make the TMR positive for both terminations as the MgO layer thickness is increased. Thus, the TMR is negative for $\mathrm{Mn}-\mathrm{Ge}$ but positive for $\mathrm{Mn}-\mathrm{Mn}$ for intermediate $\mathrm{MgO}$ thicknesses that are of interest experimentally. The balance between the native spin polarization and the Brillouin zone filtering not only accounts for the dependence of the TMR on $N_{\mathrm{MgO}}$ and the termination layer but also the bias voltage (Supplementary Fig. 7 and Supplementary Note 7).

These calculations give a natural explanation for the low TMR values found experimentally using $\mathrm{Mn}_{3} \mathrm{Ge}$ electrodes. Even though the $\mathrm{Mn}_{3} \mathrm{Ge} / \mathrm{MgO}$ interface is very smooth (Fig. 2d) inevitably there will be atomic scale fluctuations in the morphology of the $\mathrm{Mn}_{3} \mathrm{Ge}$ layer that gives rise to regions with $\mathrm{Mn}-\mathrm{Mn}$ and $\mathrm{Mn}-\mathrm{Ge}$ terminations, due to the fundamental underlying structure of the Heusler compound (see illustration in Fig. 4b). The simplest way to model such fluctuations is to average the transmission functions over the different terminations (separately for P and AP states), assuming that the $\mathrm{MgO}$ thickness is the same across the device. The TMR

Table 1 | Calculated magnetic and structural properties of $\mathrm{Mn}_{2} \mathrm{CuSb}$.

\begin{tabular}{|c|c|c|c|c|c|c|c|c|}
\hline $\begin{array}{l}\text { Heusler } \\
\text { structure }\end{array}$ & $\begin{array}{l}\text { Coupling of the } \\
\text { magnetic moments } \\
\text { of two Mn atoms }\end{array}$ & $\begin{array}{c}E_{\text {tot }} \text { in } \\
\text { cubic } \\
\text { phase } \\
\text { (eV) }\end{array}$ & $\begin{array}{c}\text { Lattice } \\
\text { constant } a_{c} \text { in } \\
\text { cubic phase } \\
(\AA)\end{array}$ & $\begin{array}{c}\text { Magnetic } \\
\text { moment in } \\
\text { cubic phase } \\
\left(\mu_{\mathrm{B}}\right)\end{array}$ & $\begin{array}{c}E_{\text {tot }} \text { in } \\
\text { tetragonal } \\
\text { phase } \\
(e V)\end{array}$ & $\begin{array}{l}\text { Lattice constant } \\
a_{t} \text { in tetragonal } \\
\text { phase }(\AA)\end{array}$ & $\begin{array}{l}\text { Lattice constant } \\
c_{t} \text { in tetragonal } \\
\text { phase }(\AA)\end{array}$ & $\begin{array}{l}\text { Magnetic } \\
\text { moment in } \\
\text { tetragonal } \\
\text { phase }\left(\mu_{\mathrm{B}}\right)\end{array}$ \\
\hline Regular & ferro & -25.111 & 6.23 & 6.4 & -25.569 & 3.86 & 7.80 & 5.4 \\
\hline Regular & ferri & -25.107 & 6.28 & 0 & \multicolumn{4}{|c|}{ No stable solution found. } \\
\hline Inverse & ferro & -24.840 & 6.12 & 4.3 & -25.292 & 3.83 & 8.19 & 6.1 \\
\hline Inverse & ferri & -25.176 & 6.22 & 1.0 & -25.272 & 3.95 & 7.82 & 0.4 \\
\hline
\end{tabular}


a

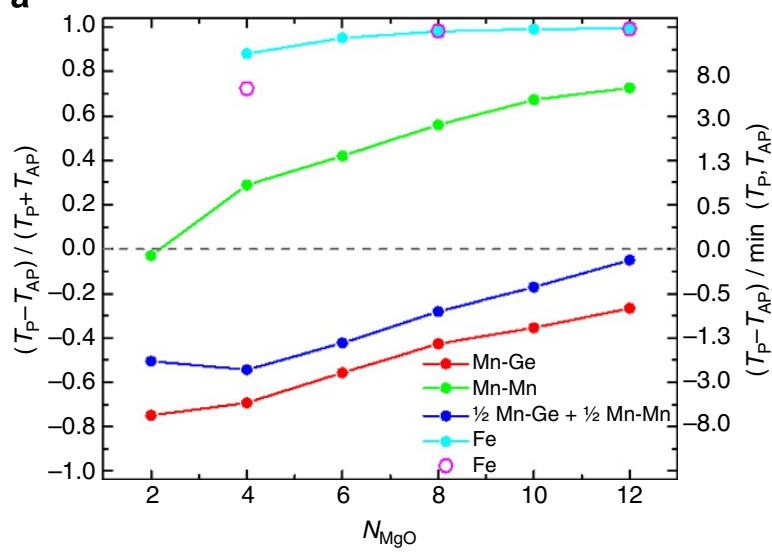

C

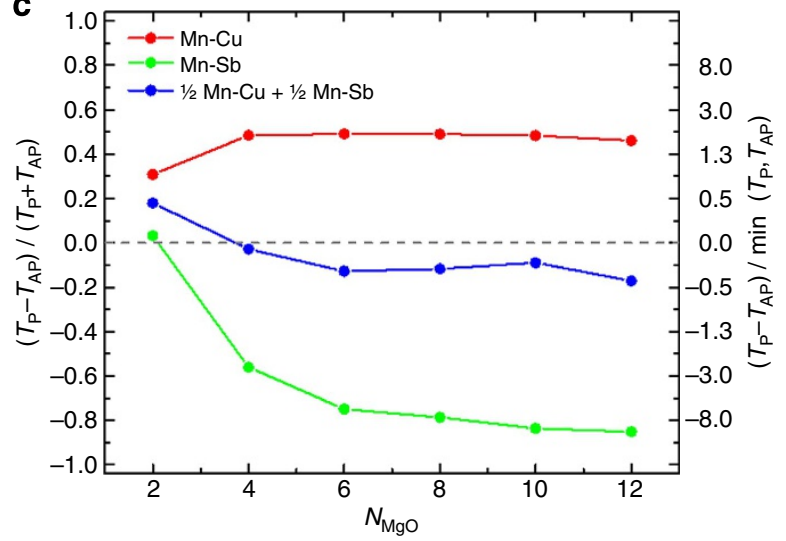

b

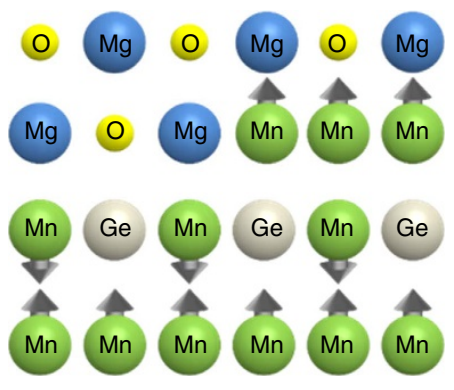

d

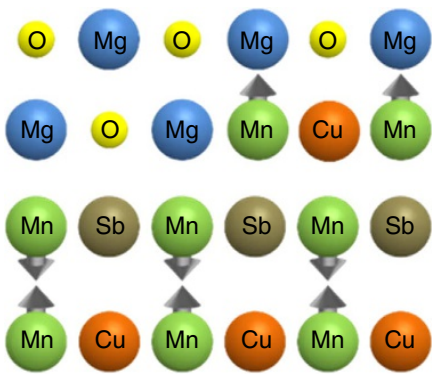

Figure 4 | Theoretical calculations of tunneling magnetoresistance. (a) TMR for $\mathrm{Mn}_{3} \mathrm{Ge} / \mathrm{MgO} / \mathrm{Fe}$ and $\mathrm{Fe} / \mathrm{MgO} / \mathrm{Fe}$ MTJs versus $\mathrm{N}_{\mathrm{MgO}}$. TMR for $\mathrm{Mn}_{3} \mathrm{Ge} / \mathrm{MgO} / \mathrm{Fe} \mathrm{MTJ}$ with $\mathrm{Mn}-\mathrm{Mn}$ termination is shown by green line, with $\mathrm{Mn}-\mathrm{Ge}$ termination by red line and for device with steps (half of the device area has $\mathrm{Mn}-\mathrm{Mn}$ termination and half of the area has $\mathrm{Mn}-\mathrm{Ge}$ termination) is shown by blue line. The TMR for Fe/MgO/Fe MTJ calculated in this work by tight-binding linear muffin-tin orbital (TB-LMTO) method is shown by cyan line and calculated by layer Korringa-Kohn-Rostoker (KKR) method (ref. 22) is shown by pink circles. (b) Schematic of atomic step between two distinct terminations with opposite magnetic moments for $\mathrm{Mn}_{3} \mathrm{Ge}$. (c) The TMR for $\mathrm{Mn}_{2} \mathrm{CuSb} / \mathrm{MgO} / \mathrm{Fe} \mathrm{MTJ}$ with $\mathrm{Mn}$-Sb termination is shown by green line, with $\mathrm{Mn}$-Cu termination by red line and for device with steps (half of the device area has $\mathrm{Mn}-\mathrm{Cu}$ termination and half of the area has $\mathrm{Mn}-\mathrm{Sb}$ termination) is shown by blue line. (d) Schematic of atomic step between two distinct terminations with opposite magnetic moments for $\mathrm{Mn}_{2} \mathrm{CuSb}$. Note that in a and $\mathbf{c}$ the TMR for the termination with the larger magnetic moment is shown in green for both $\mathrm{Mn}_{3} \mathrm{Ge}$ and $\mathrm{Mn}_{2} \mathrm{CuSb}$. In $\mathbf{b}$ and $\mathbf{d}$ the direction of the magnetic moments shown for the different termination layers for the different steps corresponds to the sign of the TMR for that termination layer (that is, arrow pointing up (down) corresponds to positive (negative) TMR).

calculated from this simple model with an assumption of equal areas occupied by $\mathrm{Mn}-\mathrm{Ge}$ and $\mathrm{Mn}-\mathrm{Mn}$ terminations is shown in Fig. 4a. The calculations give a negative TMR since both $T_{\mathrm{P}}$ and $T_{\mathrm{AP}}$ for the Mn-Ge termination are larger than those for the $\mathrm{Mn}-\mathrm{Mn}$ termination (for all $\mathrm{N}_{\mathrm{MgO}}$ considered-ranging from 2 to 12). The negative TMR is consistent with our experimental measurements. Note that due to the large $10.5 \%$ lattice mismatch between $\mathrm{MgO}$ and $\mathrm{Mn}_{3} \mathrm{Ge}$ the Brillouin zone filtering effect, which critically depends on the existence of the well-defined $2 \mathrm{D}$ Brillouin zone of the $\mathrm{Mn}_{3} \mathrm{Ge} / \mathrm{MgO}$ interface, is likely more suppressed in actual devices as compared with the large negative spin polarization effect that is less sensitive to the existence of the 2D Brillouin zone. Thus, both ideal crystal theoretical simulations (Fig. 4a) and non-ideal crystal arguments predict negative TMR for the $\mathrm{Mn}_{3} \mathrm{Ge} / \mathrm{MgO} / \mathrm{Fe}$ system, in agreement with experimental results (at low temperatures the $\mathrm{TMR}=-75 \%$ for $\sim 27 \AA$ thick MgO: Fig. 2b). We note that the surface energy of the different terminations will very likely not be identical. This could lead to the formation of a more complex surface structure but kinetic considerations will constrain the surface beyond that of the equilibrium structure.
The calculated TMR versus $\mathrm{N}_{\mathrm{MgO}}$ for $\mathrm{Mn}_{2} \mathrm{CuSb} / \mathrm{MgO} / \mathrm{Fe} \mathrm{MTJ}$, where the $\mathrm{Mn}_{2} \mathrm{CuSb}$ has a tetragonal ferrimagnetic inverse Heusler structure, with $\mathrm{Mn}-\mathrm{Sb}$ and $\mathrm{Mn}-\mathrm{Cu}$ terminations at the $\mathrm{MgO}$ interface are compared in Fig. 4c. Note that the calculated magnetic moment of the $\mathrm{Mn}-\mathrm{Sb}$ layer $\left(3.2 \mu_{\mathrm{B}}\right)$ is larger than that for the $\mathrm{Mn}-\mathrm{Cu}$ layer $\left(-2.8 \mu_{\mathrm{B}}\right)$. Interestingly, the tunnelling spin polarization from the native termination layers, that is, in the limit of $N_{\mathrm{MgO}} \rightarrow 0$, is of the opposite sign to the magnetic moment direction of these layers, whereas for $\mathrm{Mn}_{3} \mathrm{Ge}$ the spin polarization is negative for both terminations (Supplementary Fig. 8 and Supplementary Note 8). But for the range of $\mathrm{MgO}$ thicknesses of interest for technologically relevant MTJs $\left(2<N_{\mathrm{MgO}} \leq 12\right)$, tunnelling spin polarization is parallel and antiparallel, to the magnetic moment of the respective termination layer for $\mathrm{Mn}_{3} \mathrm{Ge}$ and $\mathrm{Mn}_{2} \mathrm{CuSb}$, respectively. Moreover, there is no significant Brillouin zone filtering effect for $\mathrm{Mn}_{2} \mathrm{CuSb} / \mathrm{MgO}$. In this case the dependence of the TMR on $N_{\mathrm{MgO}}$ is due to the symmetry spin filtering effect from $\mathrm{MgO} / \mathrm{Fe}$, as can be seen from Fig. 4c by comparing the calculated TMR for $\mathrm{Mn}_{2} \mathrm{CuSb} / \mathrm{MgO} / \mathrm{Fe}$, for both terminations, with that for $\mathrm{Fe} / \mathrm{MgO} / \mathrm{Fe}$. For the $\mathrm{Mn}_{2} \mathrm{CuSb} / \mathrm{MgO} /$ Fe MTJ a negative TMR is predicted for the termination layer with the largest magnetic moment $(\mathrm{Mn}-\mathrm{Sb})$ whereas for $\mathrm{Mn}_{3} \mathrm{Ge} /$ 
$\mathrm{MgO} / \mathrm{Fe}$ the opposite is the case. The TMR calculated for an MTJ with equal areas of $\mathrm{Mn}-\mathrm{Cu}$ and $\mathrm{Mn}-\mathrm{Sb}$ terminations is shown in Fig. 4c. The resulting TMR is significantly reduced due to the cancellation of contributions from the different terminations and has an overall negative sign (for $N_{\mathrm{MgO}}>2$ ). The negative sign of TMR and its small value agrees with our experimental findings.

\section{Discussion}

In summary, we have shown a method to grow highly textured ferrimagnetic Heusler films with large perpendicular anisotropy on amorphous substrates, thereby opening a path to their potential use for many applications such as magnetic recording media and rare-earth-free hard magnets. However, we find that the TMR is strongly influenced by unavoidable atomic steps at the tunnel barrier interface. When the two termination layers have opposite tunnelling spin polarizations they compensate one another leading to low TMR, as we have shown is the case for both $\mathrm{Mn}_{3} \mathrm{Ge}$ and $\mathrm{Mn}_{2} \mathrm{CuSb}$. However, the tunnelling spin polarization can be aligned either parallel, as in the case of $\mathrm{Mn}_{3} \mathrm{Ge}$, or antiparallel, as in the case of $\mathrm{Mn}_{2} \mathrm{CuSb}$, to the magnetization of the termination layers. We conclude that the most interesting and technologically useful ferrimagnetic Heusler materials will have termination layers with the same tunnelling spin polarization, that is, for one termination layer the tunnelling spin polarization is parallel to the magnetization, and for the other it is the opposite. Such materials can be identified by the use of computational techniques.

\section{Methods \\ $\mathrm{Mn}_{\mathbf{3}} \mathrm{Ge}$ and $\mathrm{Mn}_{\mathbf{2}} \mathrm{CuSb}$ film deposition and characterization. $\mathrm{Mn}_{3} \mathrm{Ge}$ and} $\mathrm{Mn}_{2} \mathrm{CuSb}$ films were deposited by d.c.-magnetron sputtering or ion-beam deposition at temperatures that were varied from ambient to $550{ }^{\circ} \mathrm{C}$. $\mathrm{Ta}_{x} \mathrm{~N}$ films were deposited with different $\mathrm{Ar} / \mathrm{N}_{2}$ ratios by reactive magnetron sputtering from a Ta target, and $200 \AA \mathrm{IrMn}_{3}$ films were deposited using ion beam sputtering from a $\mathrm{IrMn}_{3}$ target. $\mathrm{Ta}_{x} \mathrm{~N}$ and $\mathrm{IrMn}_{3}$ layers were grown at room temperature. Film compositions were measured by Rutherford back scattering measurements. X-ray diffraction measurements were carried out using a Bruker GADDS or a Bruker D8 Discover system. For the $\mathrm{Ar} / \mathrm{N}_{2}$ ratios within the range $95 / 5-75 / 25$, all $\mathrm{IrMn}_{3}$ films on $\mathrm{Ta}_{x} \mathrm{~N}$ had a preferential orientation of $(001)$. $\mathrm{IrMn}_{3}$ film grown on pure Ta underlayer showed a (111) orientation. Atomic force microscopy film characterization was made with a Bruker Icon Dimension with ScanAsyst system. High-resolution transmission electron microscopy and electron energy loss spectroscopy studies were made using a JEOL ARM 200F with a Cold-FEG source operated at $200 \mathrm{keV}$. Magnetic properties were measured at $300 \mathrm{~K}$ using a quantum design superconducting quantum interference device vibrating sample magnetometer (SQUID-VSM) in magnetic fields of up to $\pm 7 \mathrm{~T}$ in both in-plane and out-of-plane directions. Uniaxial magnetic anisotropy $K_{\mathrm{U}}$ values were calculated from $K_{\mathrm{U}}=H_{\text {eff }} \times M_{\mathrm{S}} / 2+2 \pi M_{\mathrm{S}}^{2}\left(H_{\text {eff }}\right.$ being the effective magnetic field and $M_{\mathrm{S}}$ the saturation magnetization).

Magnetic tunnel junction fabrication and characterization. MTJ devices were encapsulated in $\mathrm{Al}_{2} \mathrm{O}_{3}$. Electrical contacts were formed from $50 \AA \mathrm{Ru} / 650 \AA \mathrm{Au}$. TMR of the patterned devices was measured using a Quantum Design DynaCool physical property measurement system and a custom-built probe station with Keithley source metres 2,602 and 2,400. For fast evaluation of TMR, $R_{\mathrm{AP}}$ and $R_{\mathrm{P}}$ values in Fig. $2 \mathrm{c}$ were measured at $+0.3 \mathrm{~T}$ and $-0.3 \mathrm{~T}$, respectively, instead of sweeping the magnetic field from $\pm 9 \mathrm{~T}$.

Calculation details. The electronic structure and transmission functions of $\mathrm{Mn}_{3} \mathrm{Ge} / \mathrm{MgO} / \mathrm{Fe}, \mathrm{Mn}_{2} \mathrm{CuSb} / \mathrm{MgO} / \mathrm{Fe}$ and $\mathrm{Fe} / \mathrm{MgO} / \mathrm{Fe}$ MTJs were calculated using a tight-binding linear muffin-tin orbital method in the atomic sphere approximation with the local density approximation of DFT for the exchange-correlation energy ${ }^{17,18}$. For the $\mathrm{Mn}_{3} \mathrm{Ge} / \mathrm{MgO} / \mathrm{Fe} \mathrm{MTJ}$, the in-plane lattice constant was fixed to the experimental lattice constant $a$ of bulk tetragonal $\mathrm{Mn}_{3} \mathrm{Ge}(a=3.816 \AA$ and $c=7.261 \AA$ (ref. 19)). For $\mathrm{Mn}_{2} \mathrm{CuSb} / \mathrm{MgO} / \mathrm{Fe}$ the in-plane lattice constant was fixed to the calculated lattice constant $a$ of the $\mathrm{Mn}_{2} \mathrm{CuSb}$ tetragonal inverse ferrimagnetic phase $a=3.95 \AA$ (Table 1).

Relaxed positions of atoms at the $\mathrm{Mn}_{3} \mathrm{Ge} / \mathrm{MgO}$ and $\mathrm{Mn}_{2} \mathrm{CuSb} / \mathrm{MgO}$ interfaces (for all possible terminations) were determined using the VASP molecular dynamic program $^{20}$. The O-top configuration was found to be the most stable configuration (as compared with Mg-top and hollow) for both terminations at the $\mathrm{Mn}_{3} \mathrm{Ge} / \mathrm{MgO}$ interface (in agreement with ref. 14), and for both terminations at the $\mathrm{Mn}_{2} \mathrm{CuSb}$ / $\mathrm{MgO}$ interface. For Fe/MgO interface the atomic positions from ref. 21 were used.

\section{References}

1. Parkin, S. S. P. MgO Tunnel barriers and method of formation. US patent 8,008,097 (2003).

2. Parkin, S. S. P. et al. Giant tunneling magnetoresistance at room temperature with $\mathrm{MgO}$ (100) tunnel barriers. Nat. Mater. 3, 862-867 (2004).

3. Ikeda, S. et al. A perpendicular-anisotropy $\mathrm{CoFeB}-\mathrm{MgO}$ magnetic tunnel junction. Nat. Mater. 9, 721-724 (2010).

4. Sato, H. et al. Properties of magnetic tunnel junctions with a $\mathrm{MgO} / \mathrm{CoFeB} / \mathrm{Ta} /$ $\mathrm{CoFeB} / \mathrm{MgO}$ recording structure down to junction diameter of $11 \mathrm{~nm}$. Appl. Phys. Lett. 105, 062403 (2014).

5. Graf, T., Felser, C. \& Parkin, S. S. P. Simple rules for the understanding of Heusler compounds. Prog. Solid State Chem. 39, 1-50 (2011).

6. Balke, B., Fecher, G. H., Winterlik, J. \& Felser, C. $\mathrm{Mn}_{3} \mathrm{Ga}$, a compensated ferrimagnet with high Curie temperature and low magnetic moment for spin torque transfer applications. Appl. Phys. Lett. 90, 152504 (2007).

7. Kurt, H. et al. Magnetic and electronic properties of $\mathrm{DO}_{22}-\mathrm{Mn}_{3} \mathrm{Ge}$ (001) films. Appl. Phys. Lett. 101, 132410 (2012).

8. Sugihara, A., Mizukami, S., Yamada, Y., Koike, K. \& Miyazaki, T. High perpendicular magnetic anisotropy in $\mathrm{D}_{22}-\mathrm{Mn}_{3+\mathrm{x}} \mathrm{Ge}$ tetragonal Heusler alloy films. Appl. Phys. Lett. 104, 132404 (2014).

9. Mizukami, S. et al. Tetragonal $\mathrm{D0}_{22} \mathrm{Mn}_{3+\mathrm{x}} \mathrm{Ge}$ Epitaxial Films Grown on $\mathrm{MgO}(100)$ with a Large Perpendicular Magnetic Anisotropy. Appl. Phys. Expr 6, 123002 (2013).

10. Li, M., Jiang, X., Samant, M. G., Felser, C. \& Parkin, S. S. P. Strong dependence of the tetragonal $\mathrm{Mn}_{2.1} \mathrm{Ga}$ thin film crystallization temperature window on seed layer. Appl. Phys. Lett. 103, 032410 (2013).

11. Köhler, A., Knez, I., Ebke, D., Felser, C. \& Parkin, S. S. P. Loss of anisotropy in strained ultrathin epitaxial L1 $1_{0}$ Mn-Ga films. Appl. Phys. Lett. 103, 162406 (2013).

12. Yamaoka, T. Antiferromagnetism in $\gamma$-Phase Mn-Ir Alloys. J. Phys. Soc. Jap. 36, 445-450 (1974).

13. Winterlik, J. et al. Structural, electronic, and magnetic properties of tetragonal $\mathrm{Mn}_{3-\mathrm{x}} \mathrm{Ga}$ : Experiments and first-principles calculations. Phys. Rev. B 77, 054406 (2008).

14. Miura, Y. \& Shirai, M. Theoretical Study on Tunneling Magnetoresistance of Magnetic Tunnel Junctions with $\mathrm{DO}_{22}-\mathrm{Mn}_{3} \mathrm{Z}(\mathrm{Z}=\mathrm{Ga}, \mathrm{Ge})$. IEEE Trans. Magn 50, 1400504 (2014).

15. Zhang, D. et al. First-principles study of the structural stability of cubic, tetragonal and hexagonal phases in $\mathrm{Mn} 3 \mathrm{Z}(\mathrm{Z}=\mathrm{Ga}$, Sn and $\mathrm{Ge})$ Heusler compounds. J. Phys. Conds. Matter 25, 206006 (2013).

16. Faleev, S. V., Parkin, S. S. P. \& Mryasov, O. N. Brillouin zone spin filtering mechanism of enhanced TMR and correlation effects in $\mathrm{Co}(0001) / \mathrm{h}-\mathrm{BN} /$ $\mathrm{Co}(0001)$ magnetic tunnel junction. Preprint at http://arxiv.org/abs/1504.01017 (2015).

17. Turek, I., Drchal, V., Kudrnovsky, J., Sob, M. \& Weinberger, P. Electronic structure of disordered alloys, surfaces and interfaces (Kluwer, 1997).

18. Schilfgaarde, M. v. \& Lambrecht, W. R. L. in Tight-binding approach to computational materials science vol. 491 (eds Colombo, L., Gonis, A. \& Turchi, P.) 137 (MRS, 1998).

19. Kádár, G. \& Krén, E. Neutron diffraction study of $\mathrm{Mn}_{3}$ Ge. Int. J. Magn. 1, 143-148 (1971).

20. Kresse, G. \& Furthmüller, J. Efficient iterative schemes for ab initio total-energy calculations using a plane-wave basis set. Phys Rev. B 54, 11169-11186 (1996)

21. Wortmann, D., Bihlmayer, G. \& Blügel, S. Ab initio calculations of interface effects in tunnelling through $\mathrm{MgO}$ barriers on $\mathrm{Fe}(100)$. J. Phys. Condens. Matter 16, S5819-S5822 (2004).

22. Butler, W. H., Zhang, X.-G., Schulthess, T. C. \& MacLaren, J. M. Spindependent tunneling conductance of Fe $|\mathrm{MgO}| \mathrm{Fe}$ sandwiches. Phys. Rev. B 63, 054416 (2001).

\section{Acknowledgements}

S.V.F. acknowledges the CNMS User support by Oak Ridge National Laboratory Division of Scientific User facilities. We thank Andrew Kellock, Teya Topuria, Phil Rice, Erik Lara, Leslie Thompson, Eugene Delenia, Brian Hughes and Charles Rettner for their help.

\section{Author contributions}

J.J. and Y.F. prepared the films and performed the experiments. DFT calculations were performed by S.V.F. S.S.P.P. conceived of the study. All authors discussed the results, contributed to the analysis of the data and wrote the paper.

\section{Additional information}

Supplementary Information accompanies this paper at http://www.nature.com/ naturecommunications

Competing financial interests: The authors declare no competing financial interests. 
Reprints and permission information is available online at http://npg.nature.com/ reprintsandpermissions/

How to cite this article: Jeong, J. et al. Termination layer compensated tunnelling magnetoresistance in ferrimagnetic Heusler compounds with high perpendicular magnetic anisotropy. Nat. Commun. 7:10276 doi: 10.1038/ncomms10276 (2016). (c) (i) This work is licensed under a Creative Commons Attribution 4.0 International License. The images or other third party material in this article are included in the article's Creative Commons license, unless indicated otherwise in the credit line; if the material is not included under the Creative Commons license, users will need to obtain permission from the license holder to reproduce the material. To view a copy of this license, visit http://creativecommons.org/licenses/by/4.0/ 